Supporting Information

\title{
Quantitative Evaluation of the Activity of Low-Spin Tetravalent Nickel Ion Sites for the Oxygen Evolution Reaction
}

Yadan Ren, ${ }^{\dagger}$ Tomoya Horiguchi, ${ }^{\dagger}$ Tomoki Uchiyama, ${ }^{*} \dagger$ Yuki Orikasa, ${ }^{\ddagger}$ Toshiki Watanabe, ${ }^{\dagger}$ Kentaro Yamamoto, ${ }^{\dagger}$ Tsuyoshi Takami, ${ }^{\dagger}$ Toshiyuki Matsunaga, ${ }^{\dagger}$ Yoshinori Nishiki ${ }^{\S}$ Shigenori Mitsushima, $\|^{\perp}$ and Yoshiharu Uchimoto ${ }^{\dagger}$

${ }^{\dagger}$ Graduate School of Human and Environmental Studies, Kyoto University, Yoshida Nihonmatsu-cho, Sakyo-ku, Kyoto 606-8501, Japan

Department of Applied Chemistry, College of Life Sciences, Ritsumeikan University, 1-1-1 Noji Higashi, Kusatsu, Shiga 535-8577, Japan

${ }^{\S}$ De Nora Permelec Ltd, 2023-15 Endo, Fujisawa-shi, Kanagawa, 252-0816, Japan

"Graduate School of Engineering Science, Yokohama National University, 79-5, Tokiwadai, Hodogaya-ku, Yokohama, Kanagawa, 240-8501, Japan

${ }^{\perp}$ Institute of Advanced Sciences, Yokohama National University, 79-5, Tokiwadai, Hodogaya-ku, Yokohama, Kanagawa, 240-8501, Japan

*Email: Uchiyama.tomoki.3x@kyoto-u.ac.jp 


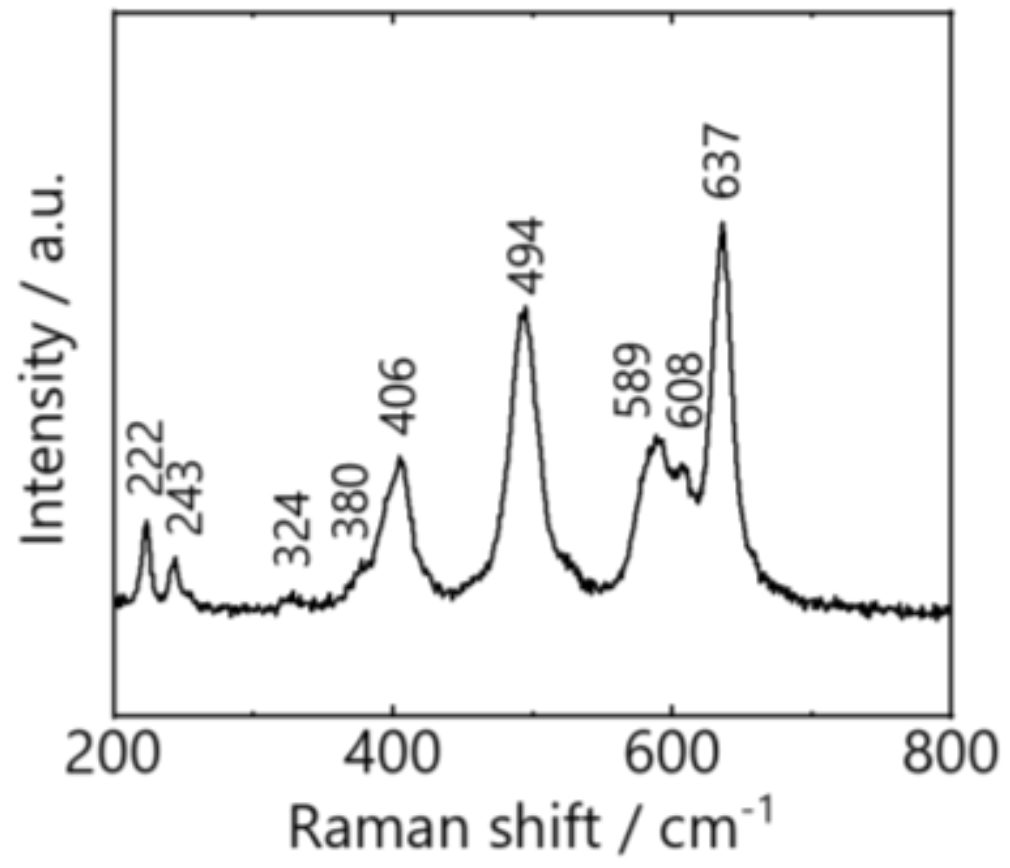

Figure S1. Raman spectrum for the pristine $\mathrm{Li}_{0.96} \mathrm{Ni}_{0.49} \mathrm{Mn}_{1.51} \mathrm{O}_{4}$. 


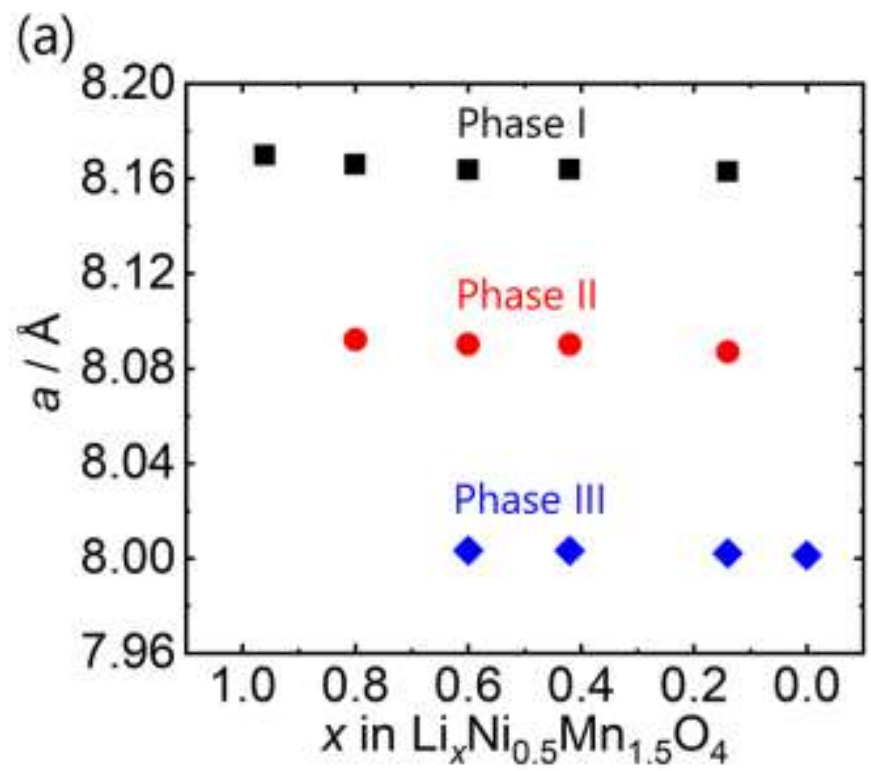

(b)

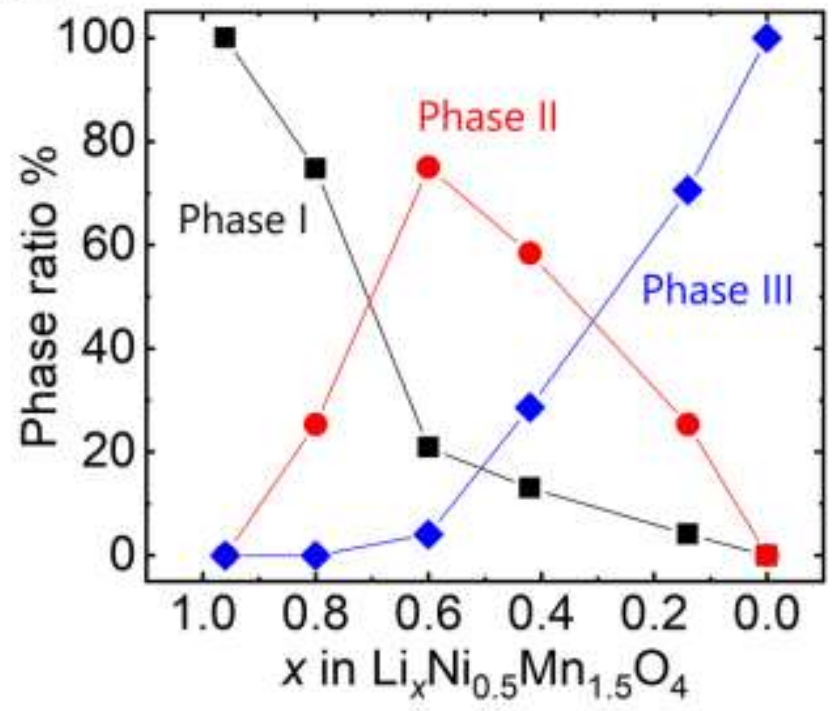

Figure S2. (a)Lattice parameters and (b) ratio of Phase I, II, III in $\mathrm{Li}_{x} \mathrm{Ni}_{0.5} \mathrm{Mn}_{1.5} \mathrm{O}_{4}$. 

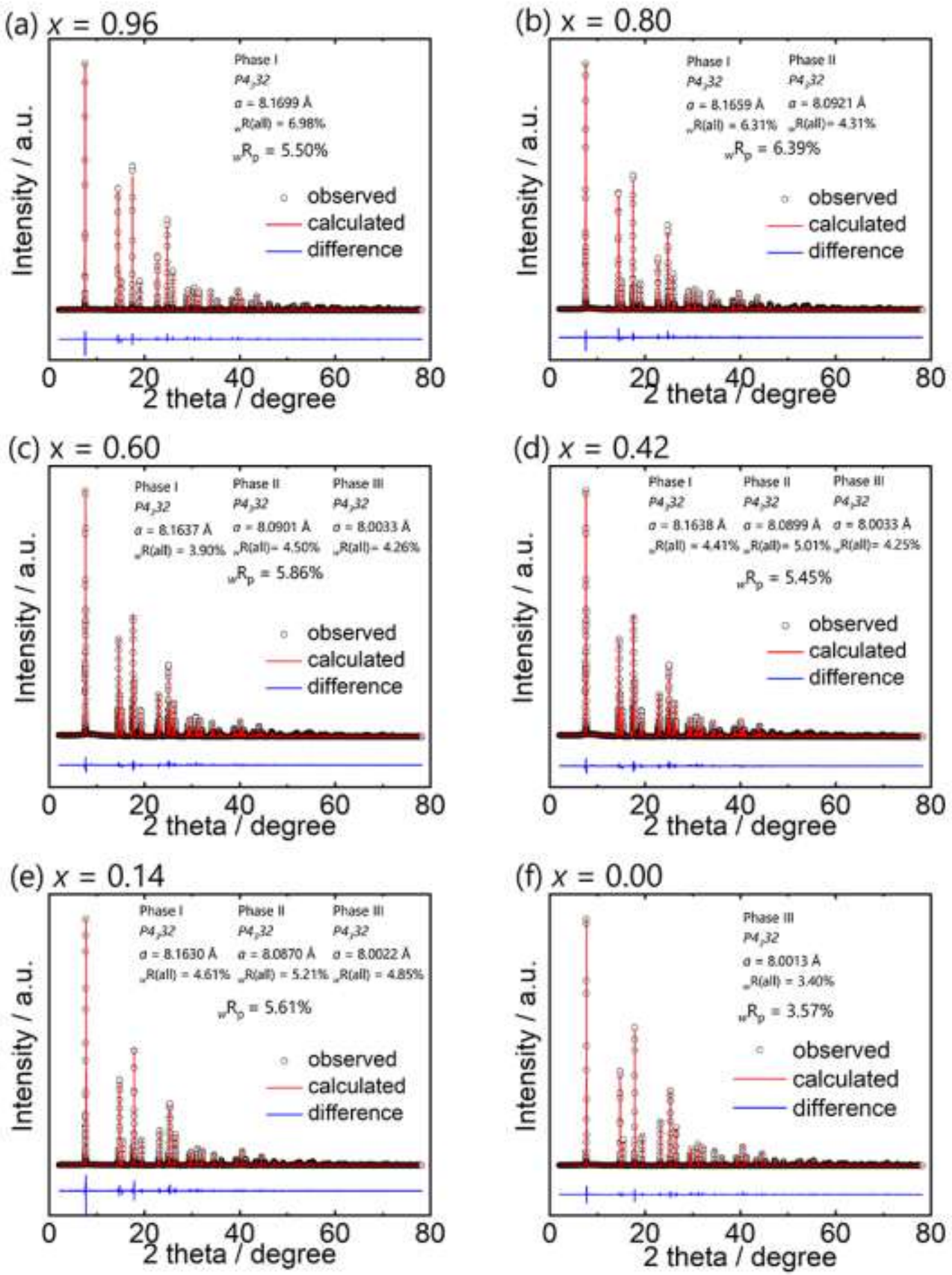

Figure S3. Rietveld refinement results for $\mathrm{Li}_{\mathrm{x}} \mathrm{Ni}_{0.5} \mathrm{Mn}_{1.5} \mathrm{O}_{4}$. 
(a) $x=0.96$

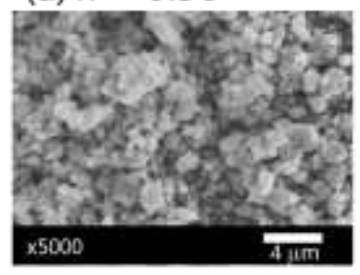

(c) $x=0.60$

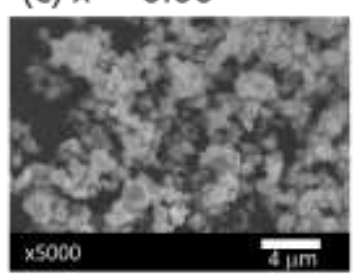

(e) $x=0.14$

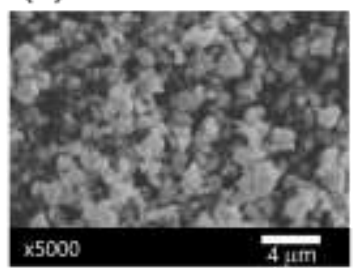

(b) $x=0.80$

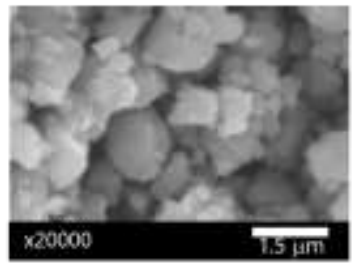

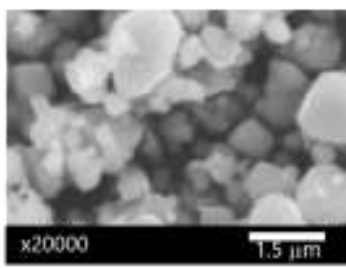

(d) $x=0.42$
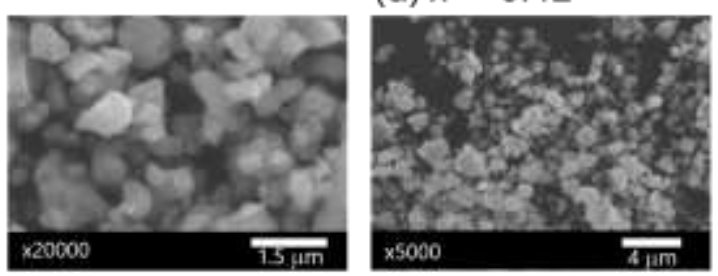

(f) $x=0.00$

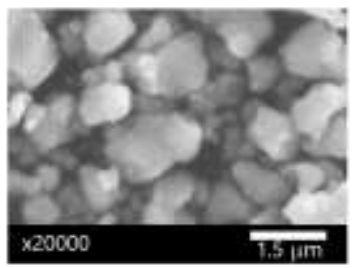

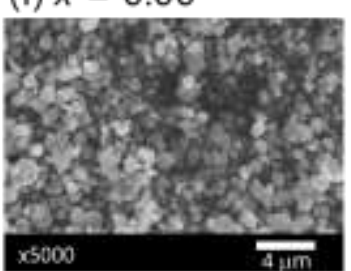

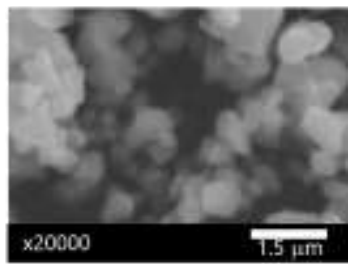

Figure S4. SEM images for $\mathrm{Li}_{\mathrm{x}} \mathrm{Ni}_{0.5} \mathrm{Mn}_{1.5} \mathrm{O}_{4}$. 

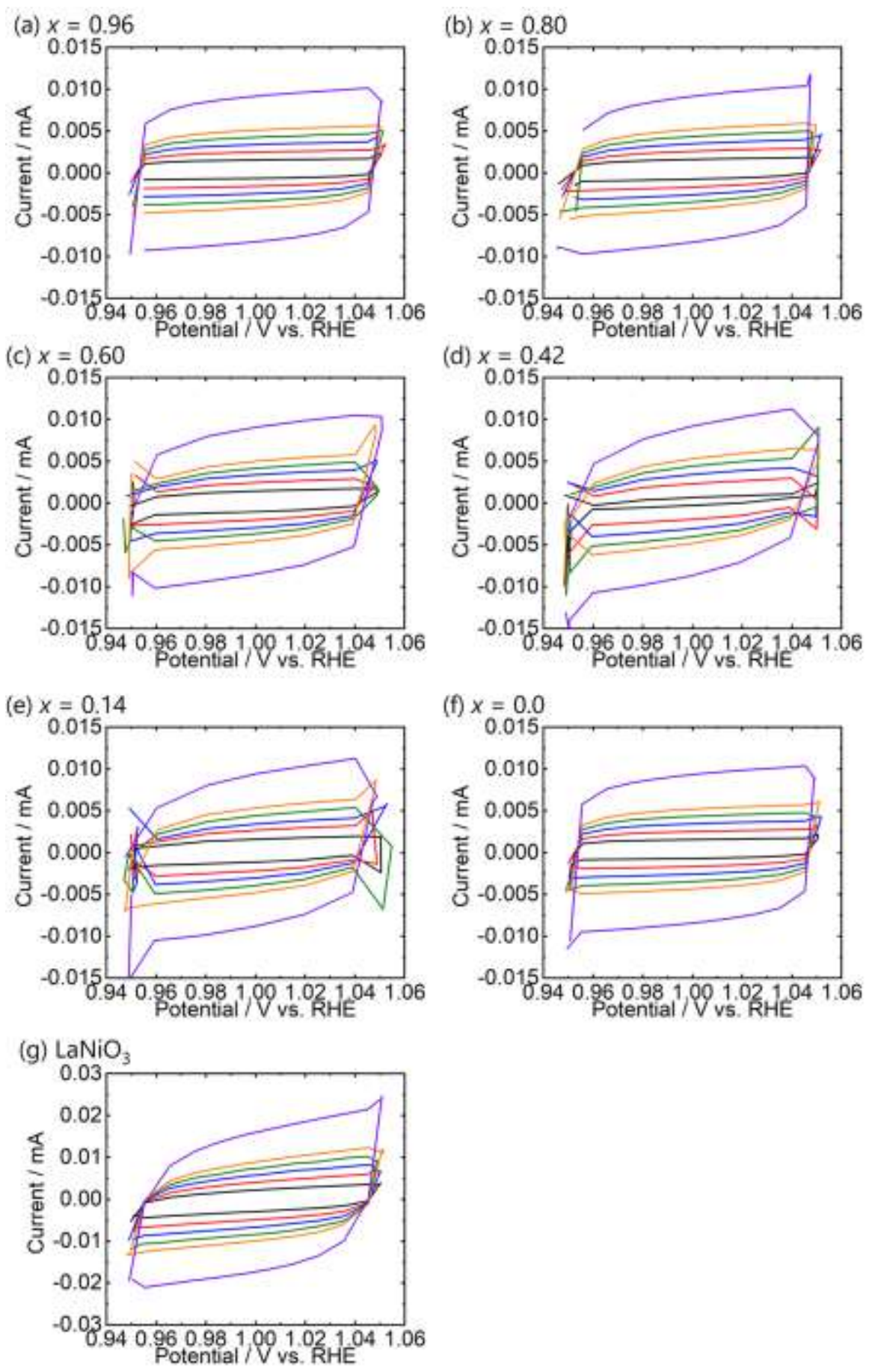

Figure S5. Double layer capacity measurement results for $\mathrm{Li}_{\mathrm{x}} \mathrm{Ni}_{0.5} \mathrm{Mn}_{1.5} \mathrm{O}_{4}$. 
(a)

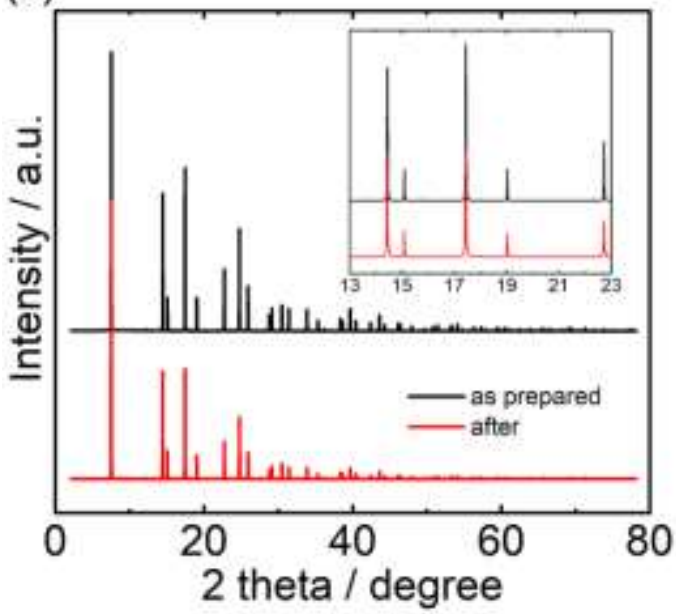

(b)

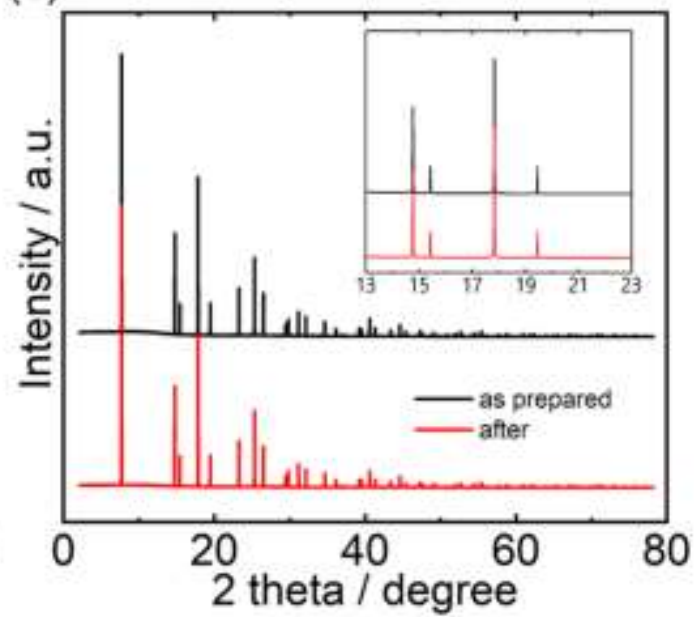

Figure S6. XRD of as prepared and after electrolysis samples for (a) $x=0.96$ and (b) $\mathrm{x}=0.0$.

(a)

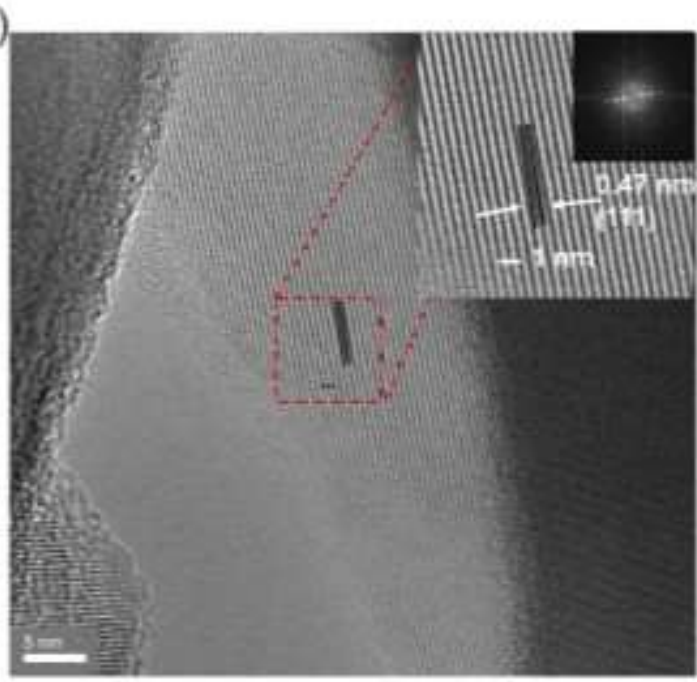

(b)

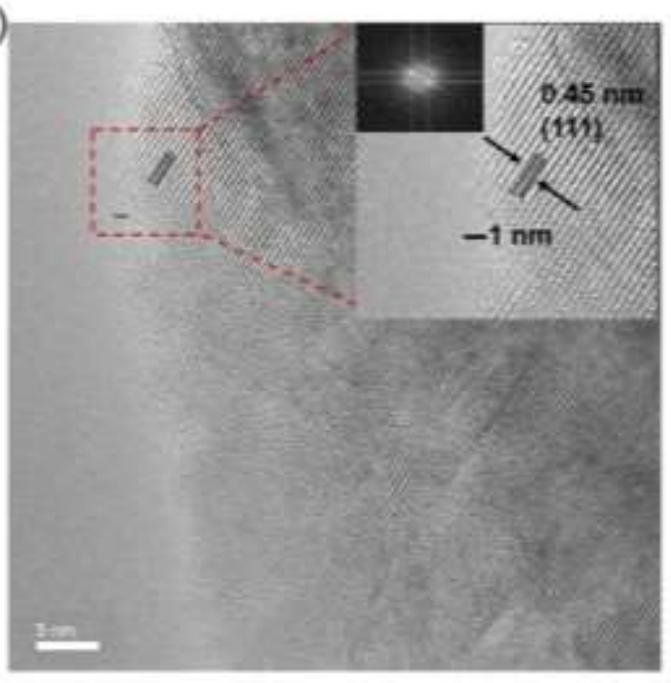

Figure S7. TEM after electrolysis for (a) $x=0.96$ and (b) $x=0.0$. 


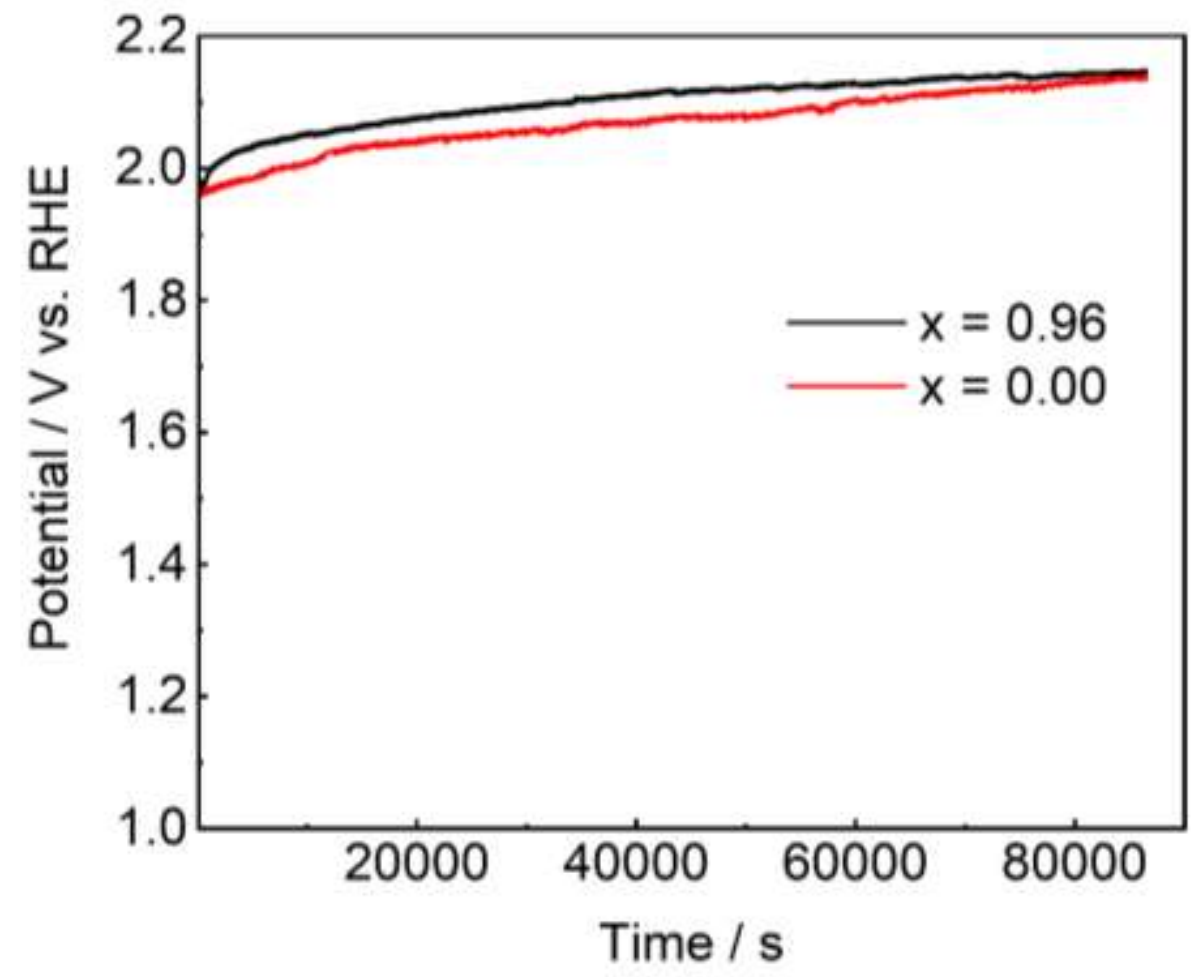

Figure S8. Long-term stability tests for $\mathrm{Li}_{0.96} \mathrm{Ni}_{0.49} \mathrm{Mn}_{1.51} \mathrm{O}_{4}$ and $\mathrm{Li}_{0.00} \mathrm{Ni}_{0.49} \mathrm{Mn}_{1.51} \mathrm{O}_{4}$. For $\mathrm{Li}_{0.00} \mathrm{Ni}_{0.49} \mathrm{Mn}_{1.51} \mathrm{O}_{4}$ sample, the current density was kept at $10 \mathrm{~mA} / \mathrm{cm}^{2}$. For $\mathrm{Li}_{0.96} \mathrm{Ni}_{0.49} \mathrm{Mn}_{1.51} \mathrm{O}_{4}$ sample, the current was kept at $6 \mathrm{~mA} / \mathrm{cm}^{2}$, making the initial potential the same for both samples before the long-term stability test. 
(a)

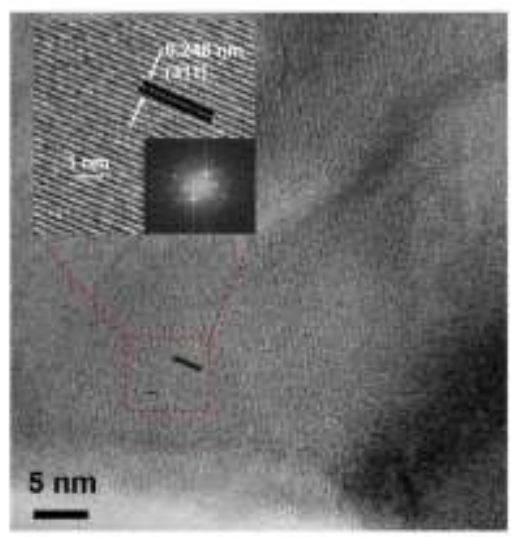

(c)

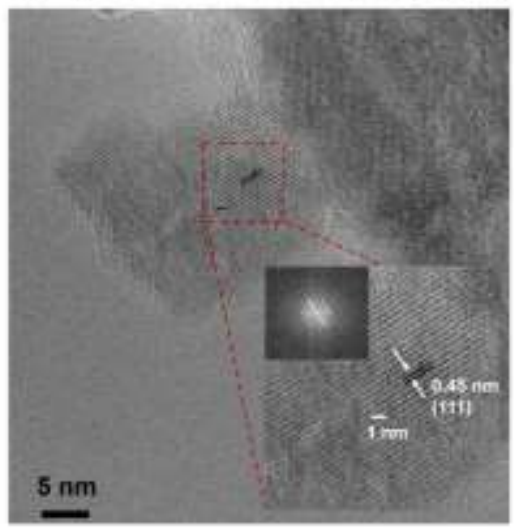

(b)

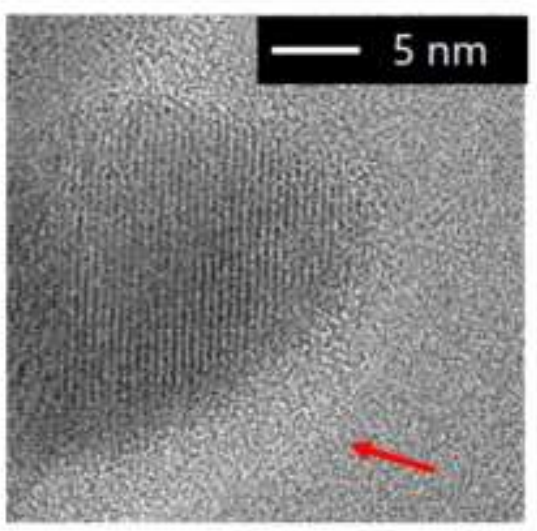

(d)

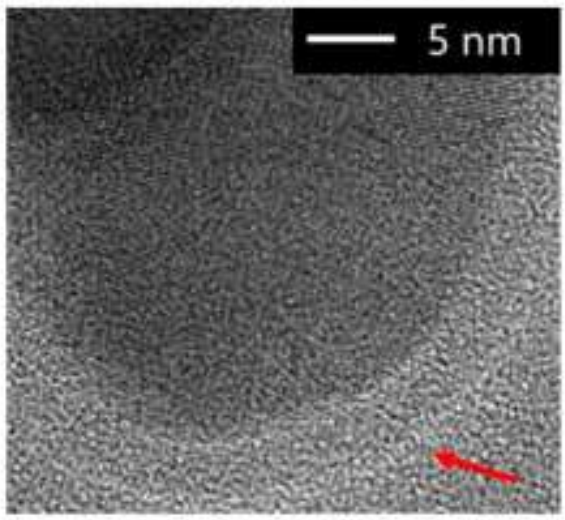

Figure S9. TEM images after long-term stability tests for (a) $\mathrm{Li}_{0.96} \mathrm{Ni}_{0.49} \mathrm{Mn}_{1.51} \mathrm{O}_{4}$ and (b) $\mathrm{Li}_{0.00} \mathrm{Ni}_{0.49} \mathrm{Mn}_{1.51} \mathrm{O}_{4}$. 


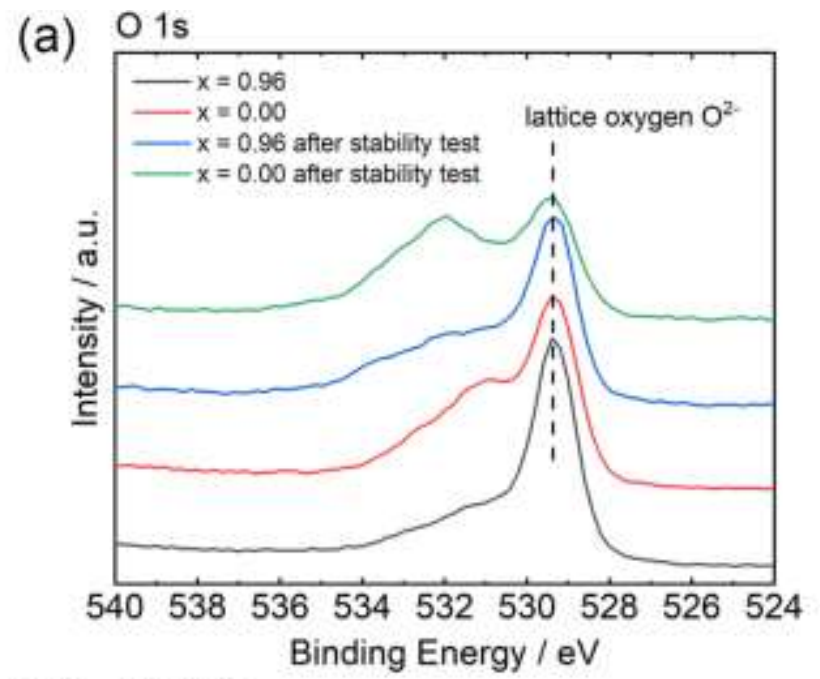

(b) $\mathrm{Ni} 2 \mathrm{p} 3 / 2$
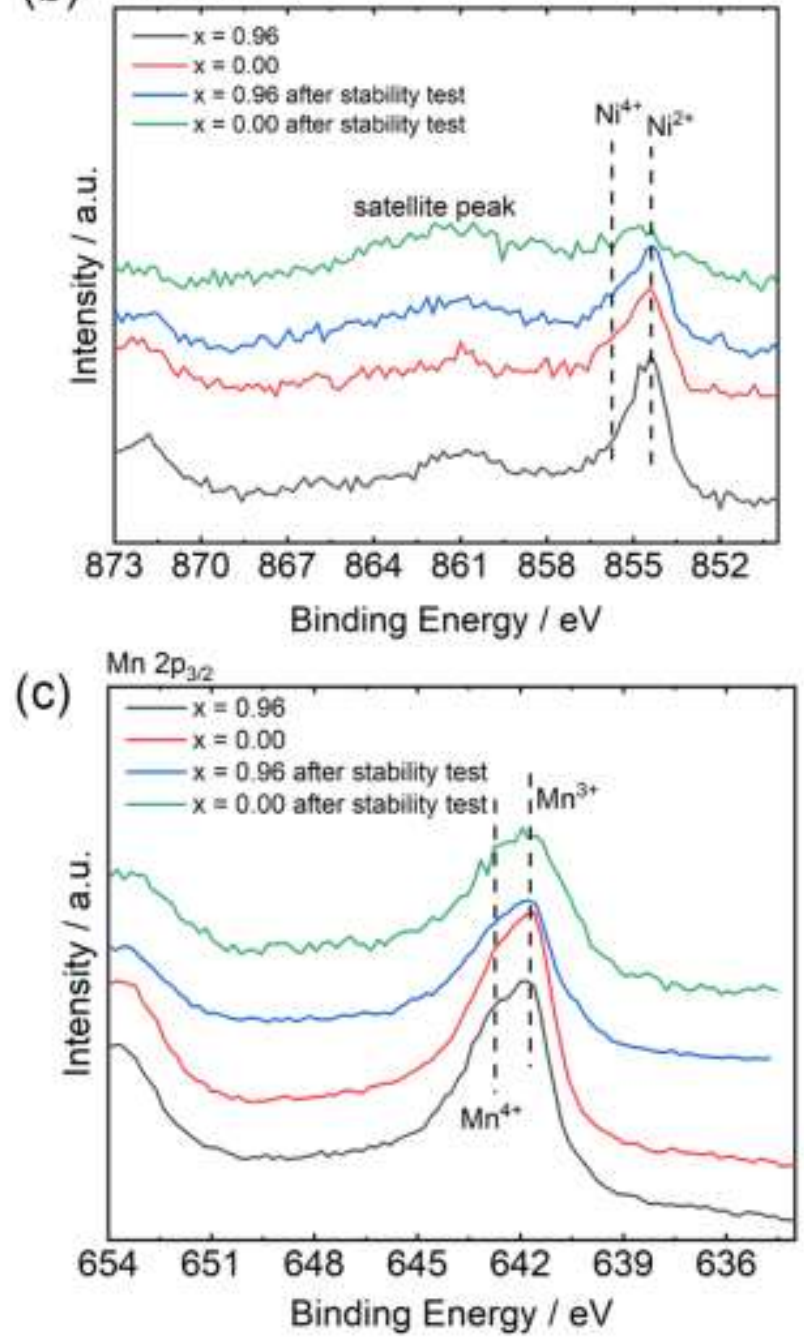

Figure S10. XPS spectra of (a) O 1s, (b) Ni 2p 3/2 and (c) Mn 2p 3/2 for $x=0.00$ and $x$ $=0.96$ before/after stability test. 
Table S1. Rietveld refinement parameters for $x=0.96$.

\begin{tabular}{|c|c|c|c|c|c|c|c|c|}
\hline Phase & Spacegroup & Atom & Wyck & S.O.F & $x / \mathrm{a}$ & $y / b$ & $z / \mathrm{c}$ & $B / \AA^{2}$ \\
\hline \multirow{5}{*}{, } & \multirow{5}{*}{$\begin{array}{r}P_{3} 32 \\
\text { - Cubic }\end{array}$} & Li1 & $8 c$ & 1.000 & 0.004 & 0.004 & 0.004 & $=\operatorname{Mn} 1(B)$ \\
\hline & & Ni1 & $4 a$ & 1.000 & 0.625 & 0.625 & 0.625 & $=\operatorname{Mn} 1(B)$ \\
\hline & & Mn1 & $12 d$ & 1.000 & 0.125 & 0.379 & -0.129 & 0.300 \\
\hline & & $\mathrm{O} 1$ & $8 c$ & 1.000 & 0.384 & 0.384 & 0.384 & $=\mathrm{O} 2(B)$ \\
\hline & & $\mathrm{O} 2$ & $24 e$ & 1.000 & 0.149 & -0.142 & 0.126 & 0.253 \\
\hline
\end{tabular}

Table S2. Rietveld refinement parameters for $x=0.80$.

\begin{tabular}{|c|c|c|c|c|c|c|c|c|c|}
\hline Phase & Spacegroup & Atom & Wyck & S.O.F & $x / \mathrm{a}$ & $y / b$ & $z / \mathrm{c}$ & $B / \AA^{2}$ & ratio \\
\hline \multirow{5}{*}{ I } & \multirow{5}{*}{$\begin{array}{r}P_{3} 32 \\
- \text { Cubic }\end{array}$} & Li1 & $8 c$ & 1.000 & 0.004 & 0.004 & 0.004 & $=\operatorname{Mn} 1(B)$ & \multirow{5}{*}{$74.70 \%$} \\
\hline & & Ni1 & $4 a$ & 1.000 & 0.625 & 0.625 & 0.625 & $=\operatorname{Mn} 1(B)$ & \\
\hline & & Mn1 & $12 d$ & 1.000 & 0.125 & 0.378 & -0.128 & 0.325 & \\
\hline & & $\mathrm{O} 1$ & $8 c$ & 1.000 & 0.389 & 0.389 & 0.389 & $=\mathrm{O} 2(B)$ & \\
\hline & & $\mathrm{O} 2$ & $24 e$ & 1.000 & 0.140 & -0.144 & 0.121 & 0.273 & \\
\hline \multirow{5}{*}{ II } & \multirow{5}{*}{$\begin{array}{r}P_{3} 32 \\
- \text { Cubic }\end{array}$} & Li1 & $8 c$ & 0.500 & 0.004 & 0.004 & 0.004 & $=\operatorname{Mn} 1(B)$ & \multirow{5}{*}{$25.30 \%$} \\
\hline & & Ni1 & $4 a$ & 1.000 & 0.625 & 0.625 & 0.625 & $=\operatorname{Mn} 1(B)$ & \\
\hline & & Mn1 & $12 d$ & 1.000 & 0.125 & 0.379 & -0.129 & 0.324 & \\
\hline & & $\mathrm{O} 1$ & $8 c$ & 1.000 & 0.384 & 0.384 & 0.384 & $=\mathrm{O} 2(B)$ & \\
\hline & & $\mathrm{O} 2$ & $24 e$ & 1.000 & 0.133 & -0.142 & 0.126 & 0.271 & \\
\hline
\end{tabular}


Table S3. Rietveld refinement parameters for $x=0.60$.

\begin{tabular}{|c|c|c|c|c|c|c|c|c|c|}
\hline Phase & Spacegroup & Atom & Wyck & S.O.F & $x / \mathrm{a}$ & $y / \mathrm{b}$ & $z / \mathrm{c}$ & $B / \AA^{2}$ & ratio \\
\hline \multirow{5}{*}{ I } & \multirow{5}{*}{$\begin{array}{r}P 4_{3} 32 \\
- \text { Cubic }\end{array}$} & Li1 & $8 c$ & 1.000 & 0.004 & 0.004 & 0.004 & $=\operatorname{Mn} 1(B)$ & \multirow{5}{*}{$20.90 \%$} \\
\hline & & Ni1 & $4 a$ & 1.000 & 0.625 & 0.625 & 0.625 & $=\operatorname{Mn} 1(B)$ & \\
\hline & & Mn1 & $12 d$ & 1.000 & 0.125 & 0.378 & -0.128 & 0.326 & \\
\hline & & $\mathrm{O} 1$ & $8 c$ & 1.000 & 0.389 & 0.389 & 0.389 & $=\mathrm{O} 2(B)$ & \\
\hline & & $\mathrm{O} 2$ & $24 e$ & 1.000 & 0.140 & -0.144 & 0.121 & 0.274 & \\
\hline \multirow{5}{*}{ II } & \multirow{5}{*}{$\begin{array}{r}\mathrm{P}_{3} 32 \\
- \text { Cubic }\end{array}$} & Li1 & $8 c$ & 0.500 & 0.004 & 0.004 & 0.004 & $=\operatorname{Mn} 1(B)$ & \multirow{5}{*}{$75.01 \%$} \\
\hline & & Ni1 & $4 a$ & 1.000 & 0.625 & 0.625 & 0.625 & $=\operatorname{Mn} 1(B)$ & \\
\hline & & Mn1 & $12 d$ & 1.000 & 0.125 & 0.377 & -0.127 & 0.324 & \\
\hline & & $\mathrm{O} 1$ & $8 c$ & 1.000 & 0.386 & 0.386 & 0.386 & $=\mathrm{O} 2(B)$ & \\
\hline & & $\mathrm{O} 2$ & $24 e$ & 1.000 & 0.142 & -0.143 & 0.126 & 0.251 & \\
\hline \multirow{5}{*}{ III } & \multirow{5}{*}{$\begin{array}{r}\mathrm{P}_{3} 32 \\
- \text { Cubic }\end{array}$} & Li1 & $8 c$ & 0.000 & 0.004 & 0.004 & 0.004 & $=\operatorname{Mn} 1(B)$ & \multirow{5}{*}{$4.09 \%$} \\
\hline & & Ni1 & $4 a$ & 1.000 & 0.625 & 0.625 & 0.625 & $=\operatorname{Mn} 1(B)$ & \\
\hline & & Mn1 & $12 d$ & 1.000 & 0.125 & 0.375 & -0.125 & 0.328 & \\
\hline & & $\mathrm{O} 1$ & $8 c$ & 1.000 & 0.388 & 0.388 & 0.388 & $=\mathrm{O} 2(B)$ & \\
\hline & & $\mathrm{O} 2$ & $24 e$ & 1.000 & 0.137 & -0.142 & 0.132 & 0.291 & \\
\hline
\end{tabular}


Table S4. Rietveld refinement parameters for $x=0.40$.

\begin{tabular}{|c|c|c|c|c|c|c|c|c|c|}
\hline Phase & Spacegroup & Atom & Wyck & S.O.F & $x / \mathrm{a}$ & $y / b$ & $z / c$ & $B / \AA^{2}$ & ratio \\
\hline \multirow{5}{*}{ I } & \multirow{5}{*}{$\begin{array}{r}P_{3} 32 \\
- \text { Cubic }\end{array}$} & Li1 & $8 c$ & 1.000 & 0.005 & 0.005 & 0.005 & $=\operatorname{Mn} 1(B)$ & \multirow{5}{*}{$13.00 \%$} \\
\hline & & Ni1 & $4 a$ & 1.000 & 0.625 & 0.625 & 0.625 & $=\operatorname{Mn} 1(B)$ & \\
\hline & & Mn1 & $12 d$ & 1.000 & 0.125 & 0.378 & -0.128 & 0.325 & \\
\hline & & $\mathrm{O} 1$ & $8 c$ & 1.000 & 0.389 & 0.389 & 0.389 & $=\mathrm{O} 2(B)$ & \\
\hline & & $\mathrm{O} 2$ & $24 e$ & 1.000 & 0.140 & -0.144 & 0.121 & 0.281 & \\
\hline \multirow{5}{*}{ II } & \multirow{5}{*}{$\begin{array}{r}\mathrm{P}_{3} 32 \\
- \text { Cubic }\end{array}$} & Li1 & $8 c$ & 0.500 & 0.004 & 0.004 & 0.004 & $=\operatorname{Mn} 1(B)$ & \multirow{5}{*}{$58.40 \%$} \\
\hline & & Ni1 & $4 a$ & 1.000 & 0.625 & 0.625 & 0.625 & $=\operatorname{Mn} 1(B)$ & \\
\hline & & Mn1 & $12 d$ & 1.000 & 0.125 & 0.377 & -0.127 & 0.324 & \\
\hline & & $\mathrm{O} 1$ & $8 c$ & 1.000 & 0.386 & 0.386 & 0.386 & $=\mathrm{O} 2(B)$ & \\
\hline & & $\mathrm{O} 2$ & $24 e$ & 1.000 & 0.142 & -0.143 & 0.128 & 0.276 & \\
\hline \multirow{5}{*}{ III } & \multirow{5}{*}{$\begin{array}{r}P_{3} 32 \\
- \text { Cubic }\end{array}$} & Li1 & $8 c$ & 0.000 & 0.004 & 0.004 & 0.004 & $=\operatorname{Mn} 1(B)$ & \multirow{5}{*}{$28.60 \%$} \\
\hline & & Ni1 & $4 a$ & 1.000 & 0.625 & 0.625 & 0.625 & $=\operatorname{Mn} 1(B)$ & \\
\hline & & Mn1 & $12 d$ & 1.000 & 0.125 & 0.375 & -0.125 & 0.327 & \\
\hline & & $\mathrm{O} 1$ & $8 c$ & 1.000 & 0.388 & 0.388 & 0.388 & $=\mathrm{O} 2(B)$ & \\
\hline & & $\mathrm{O} 2$ & $24 e$ & 1.000 & 0.137 & -0.140 & 0.132 & 0.297 & \\
\hline
\end{tabular}


Table S5. Rietveld refinement parameters for $x=0.14$.

\begin{tabular}{|c|c|c|c|c|c|c|c|c|c|}
\hline Phase & Spacegroup & Atom & Wyck & S.O.F & $x / \mathrm{a}$ & $y / \mathrm{b}$ & $z / \mathrm{c}$ & $B / \AA^{2}$ & ratio \\
\hline \multirow{5}{*}{ I } & \multirow{5}{*}{$\begin{array}{r}P 4_{3} 32 \\
- \text { Cubic }\end{array}$} & Li1 & $8 c$ & 1.000 & -0.000 & -0.000 & -0.000 & $=\operatorname{Mn} 1(B)$ & \multirow{5}{*}{$4.08 \%$} \\
\hline & & Ni1 & $4 a$ & 1.000 & 0.625 & 0.625 & 0.625 & $=\operatorname{Mn} 1(B)$ & \\
\hline & & Mn1 & $12 d$ & 1.000 & 0.125 & 0.378 & -0.128 & 0.315 & \\
\hline & & $\mathrm{O} 1$ & $8 c$ & 1.000 & 0.389 & 0.389 & 0.389 & $=\mathrm{O} 2(B)$ & \\
\hline & & $\mathrm{O} 2$ & $24 e$ & 1.000 & 0.140 & -0.144 & 0.121 & 0.276 & \\
\hline \multirow{5}{*}{ II } & \multirow{5}{*}{$\begin{array}{r}\mathrm{P}_{3} 32 \\
- \text { Cubic }\end{array}$} & Li1 & $8 c$ & 0.500 & 0.004 & 0.004 & 0.004 & $=\operatorname{Mn} 1(B)$ & \multirow{5}{*}{$25.31 \%$} \\
\hline & & Ni1 & $4 a$ & 1.000 & 0.625 & 0.625 & 0.625 & $=\operatorname{Mn} 1(B)$ & \\
\hline & & Mn1 & $12 d$ & 1.000 & 0.125 & 0.377 & -0.127 & 0.322 & \\
\hline & & $\mathrm{O} 1$ & $8 c$ & 1.000 & 0.386 & 0.386 & 0.386 & $=\mathrm{O} 2(B)$ & \\
\hline & & $\mathrm{O} 2$ & $24 e$ & 1.000 & 0.142 & -0.143 & 0.128 & 0.288 & \\
\hline \multirow{5}{*}{ III } & \multirow{5}{*}{$\begin{array}{r}P 4_{3} 32 \\
- \text { Cubic }\end{array}$} & Li1 & $8 c$ & 0.000 & 0.004 & 0.004 & 0.004 & $=\operatorname{Mn} 1(B)$ & \multirow{5}{*}{$70.61 \%$} \\
\hline & & Ni1 & $4 a$ & 1.000 & 0.625 & 0.625 & 0.625 & $=\operatorname{Mn} 1(B)$ & \\
\hline & & Mn1 & $12 d$ & 1.000 & 0.125 & 0.375 & -0.125 & 0.325 & \\
\hline & & $\mathrm{O} 1$ & $8 c$ & 1.000 & 0.388 & 0.388 & 0.388 & $=\mathrm{O} 2(B)$ & \\
\hline & & $\mathrm{O} 2$ & $24 e$ & 1.000 & 0.137 & -0.140 & 0.132 & 0.256 & \\
\hline
\end{tabular}

Table S6. Rietveld refinement parameters for $x=0.0$.

\begin{tabular}{|c|c|c|c|c|c|c|c|c|}
\hline Phase & Spacegroup & Atom & Wyck & S.O.F & $x / \mathrm{a}$ & $y / b$ & $z / c$ & $B / \AA^{2}$ \\
\hline \multirow{5}{*}{ III } & \multirow{5}{*}{$\begin{array}{r}\mathrm{P}_{3} 32 \\
- \text { - Cubic }\end{array}$} & Li1 & $8 c$ & 0.000 & 0.004 & 0.004 & 0.004 & $=\operatorname{Mn} 1(B)$ \\
\hline & & Ni1 & $4 a$ & 1.000 & 0.625 & 0.625 & 0.625 & $=\operatorname{Mn} 1(B)$ \\
\hline & & Mn1 & $12 d$ & 1.000 & 0.125 & 0.375 & -0.125 & 0.312 \\
\hline & & $\mathrm{O} 1$ & $8 c$ & 1.000 & 0.391 & 0.391 & 0.391 & $=\mathrm{O} 2(B)$ \\
\hline & & $\mathrm{O} 2$ & $24 e$ & 1.000 & 0.135 & -0.138 & 0.137 & 0.156 \\
\hline
\end{tabular}


Table S7. The electrochemical surface area parameters for $\mathrm{Li}_{x} \mathrm{Ni}_{0.5} \mathrm{Mn}_{1.5} \mathrm{O}_{4}$.

\begin{tabular}{ccc} 
& $C_{D L} / m F$ & $\mathrm{ECSA} / \mathrm{cm}^{2}$ \\
\hline$x=0.96$ & 0.043 & 1.1 \\
$x=0.80$ & 0.043 & 1.1 \\
$x=0.60$ & 0.042 & 1.1 \\
$x=0.42$ & 0.042 & 1.1 \\
$x=0.14$ & 0.043 & 1.1 \\
$x=0.0$ & 0.043 & 1.1 \\
$\mathrm{LaNiO}_{3}$ & 0.078 & 2.0 \\
\hline
\end{tabular}

Table S8. Tafel slope value of $\mathrm{Li}_{x} \mathrm{Ni}_{0.5} \mathrm{Mn}_{1.5} \mathrm{O}_{4}$ series and $\mathrm{LaNiO}_{3}$.

\begin{tabular}{cc}
\hline & Tafel slope $/ \mathrm{mV} \mathrm{dec}^{-1}$ \\
\hline$x=0.96$ & 121.9 \\
$x=0.80$ & 98.2 \\
$x=0.60$ & 89.2 \\
$x=0.42$ & 92.4 \\
$x=0.14$ & 75.3 \\
$x=0.0$ & 66.0 \\
$\mathrm{LaNiO}_{3}$ & 73.3 \\
\hline
\end{tabular}

